\title{
On Fefferman's Non-existence Problems
}

\author{
N. Aliev, Sh. Rezapour, and M. Jahanshahi
}

\begin{abstract}
Fefferman has propounded four open Cauchy problems A, B, C and D in Navier-Stokes equations. The problems A and B refer to sufficient condition for the existence of solutions, and the problems $\mathrm{C}$ and $\mathrm{D}$ to the ones for the non-existence of solutions. Here, we shall answer to the problems $\mathrm{C}$ and D.
\end{abstract}

\section{INTRODUCTION}

The Euler and Navier-Stokes equations describe the motion of a fluid in $\mathbb{R}^{n}$ $(\mathrm{n}=2$ or $\mathrm{n}=3)([2],[5])$. The steady-state of 3-dimensional case of Navier-Stokes system of equations has been considered as a boundary value problem (BVP) in [1]. This BVP for stationary case is associated to flows with free surfaces, flows around bodies, channels and wakes behind bodies. In all these problems NavierStokes equations are investigated over (finite or infinite) domain with boundary conditions determined by physical considerations. But for non-stationary problems one needs besides the boundary conditions suitable initial conditions. In this cases the Navier-Stokes equations are to be solved for an unknown velocity vector $u(x, t)=\left(u_{i}(x, t)\right)_{1 \leq i \leq n} \in \mathbb{R}^{n}$ and pressure $p(x, t) \in \mathbb{R}$, defined for space-variable $x \in \mathbb{R}^{n}$ and time $t \geq 0$. We restrict attention here to incompressible fluids filling all of $\mathbf{R}^{n}$. The Navier-Stokes equations are then given by

$$
\begin{aligned}
& \frac{\partial u_{i}}{\partial t}+\sum_{j=1}^{n} u_{j} \frac{\partial u_{i}}{\partial x_{j}}=\nu \Delta u_{i}-\frac{\partial p}{\partial x_{i}}+f_{i}(x, t) \quad\left(x \in \mathbf{R}^{n}, t \geq 0\right) \\
& \operatorname{div} u=\sum_{i=1}^{n} \frac{\partial u_{i}}{\partial x_{i}}=0 \quad\left(x \in \mathbb{R}^{n}, t \geq 0\right)
\end{aligned}
$$

with initial conditions

$$
u(x, 0)=u^{0}(x) \quad\left(x \in \mathbb{R}^{n}\right) .
$$

Here, $u^{0}(x)$ is a given $C^{\infty}$ divergence-free vector field on $\mathbb{R}^{n}, f_{i}(x, t)$ are the components of a given externally applied force (e.g. gravity), $\nu$ is a positive coefficient (the viscosity) and $\Delta=\sum_{i=1}^{n} \frac{\partial^{2}}{\partial x_{i}^{2}}$ is the Laplacian in the space variables.

2000 Mathematics Subject Classification. Primary: 35K15, 35K45.

Key words and phrases. Navier-Stokes equations, Non-existence Problems, Periodic Function. 
Equation (1) is just Newton's gravity low, that is $f=m a$, for a fluid element subject to the external force $f=\left(f_{i}(x, t)\right)_{1 \leq i \leq n}$ and to the forces arising from pressure and friction. Equation (2) just says that fluid is incompressible. For physically requirements, we want to make sure $u(x, t)$ does now grow large as $|x| \longrightarrow \infty$. Hence, we will restrict to forces $f$ and initial conditions $u^{0}$ that satisfy

$$
\left|\partial_{x}^{\alpha} u^{0}(x)\right| \leq C_{\alpha K}(1+|x|)^{-K} \text { on } \mathbf{R}^{n} \text {, for any } \alpha \text { and } K
$$

and

$$
\left|\partial_{x}^{\alpha} \partial_{t}^{m} f(x, t)\right| \leq C_{\alpha m K}(1+|x|+t)^{-K} \text { on } \mathbb{R}^{n} \times[0, \infty) \text {, for any } \alpha, m, K .
$$

For physical considerations we suppose a solution of (1), (2) and (3) which satisfies

$$
p, u \in C^{\infty}\left(\mathbb{R}^{n} \times[0, \infty)\right)
$$

and

$$
\int_{\mathbb{R}^{n}}|u(x, t)|^{2} \mathrm{~d} x<C, \quad \text { for all } t \geq 0 \text { (boundary energy). }
$$

Consequently, to rule out problems at infinity, we may look for spatially periodic solutions of (1), (2) and (3). Thus, we assume that $u^{0}(x), f(x, t)$ satisfy the following periodic conditions

$$
u^{0}\left(x+e_{j}\right)=u^{0}(x), \quad f\left(x+e_{j}, t\right)=f(x, t), \quad 1 \leq j \leq n
$$

$\left(e_{j}\right.$ is the $j$-th unit vector in $\left.\mathbb{R}^{n}\right)$.

In place of (4) and (5), we assume that $u^{0}$ is a smooth function, and that

$$
\left|\partial_{x}^{\alpha} \partial_{t}^{m} f(x, t)\right| \leq C_{\alpha m K}(1+|t|)^{-K} \text { on } \mathbb{R}^{n} \times[0, \infty) \text {, for any } \alpha, m, K \text {. }
$$

We then accept a solution of (1), (2) and (3) as physically reasonable if it satisfies

$$
u\left(x+e_{j}, t\right)=u(x, t), \text { on } \mathbb{R}^{n} \times[0, \infty), \text { and for } 1 \leq j \leq n
$$

and

$$
p, u \in C^{\infty}\left(\mathbf{R}^{n} \times[0, \infty)\right) .
$$

A fundamental problem in analysis is to decide whether such smooth, physically reasonable solutions exist for the Navier-Stokes equations ([3]-[5]). To give reasonable leeway to solvers while retaining the heart of the problem, Fefferman asked for a proof of one the following four statements ([2], [4]).

(A) Existence and Smoothness of Navier-Stokes Solutions on $\mathbb{R}^{3}$. Take $\nu>0$ and $n=3$. Let $u^{0}(x)$ be any smooth, divergence-free vector field satisfying (4). Take $f(x, t)$ to be identically zero. Then there exist smooth functions $p(x, t)$, $u_{i}(x, t)$ on $\mathbf{R}^{3} \times[0, \infty)$ that satisfy (1), (2), (3), (6) and (7).

(B) Existence and Smoothness of Navier-Stokes Solutions in $\mathbb{R}^{3} / \mathbf{Z}^{3}$. Take $\nu>0$ and $n=3$. Let $u^{0}(x)$ be any smooth, divergence-free vector field satisfying (8). Take $f(x, t)$ to be identically zero. Then there exist smooth functions $p(x, t), u_{i}(x, t)$ on $\mathbb{R}^{3} \times[0, \infty)$ that satisfy (1), (2), (3), (10) and (11). 
(C) Breakdown of Navier-Stokes Solutions on $\mathbb{R}^{3}$. Take $\nu>0$ and $n=3$. Then there exist a smooth, divergence-free vector field $u^{0}(x)$ on $\mathbf{R}^{3}$ and a smooth, $f(x, t)$ on $\mathbf{R}^{3} \times[0, \infty)$ satisfying (4) and (5), for which there exist no solutions $(p, u)$ of (1), (2), (3), (6) and (7) on $\mathbf{R}^{3} \times[0, \infty)$.

(D) Breakdown of Navier-Stokes Solutions on $\mathbb{R}^{3} / \mathbf{Z}^{3}$. Take $\nu>0$ and $n=3$. Then there exist a smooth, divergence-free vector field $u^{0}(x)$ on $\mathbb{R}^{3}$ and a smooth, $f(x, t)$ on $\mathbf{R}^{3} \times[0, \infty)$ satisfying (8) and (9), for which there exist no solutions $(p, u)$ of (1), (2), (3), (10) and (11) on $\mathbf{R}^{3} \times[0, \infty)$.

\section{The Problem C}

In this section we shall give some sufficient conditions for non-existence of solutions of the problem C.

Theorem 2.1. If there exists an index $i(1 \leq i \leq 3)$ such that

$$
\int_{\mathbb{R}^{3}} \mathrm{~d} x\left[\int_{0}^{\infty} f_{i}(x, t) \mathrm{d} t+u_{i}^{0}(x)\right] \neq 0,
$$

then the problem $C$ holds.

Proof. For $x \in \mathbb{R}^{3}$ and $t \geq 0$, we reform (1) in the following integral equation

$$
\begin{aligned}
& \int_{\mathbb{R}^{3}} \mathrm{~d} x \int_{0}^{\infty} \frac{\partial u_{i}(x, t)}{\partial t} \mathrm{~d} t+\sum_{j=1}^{3} \int_{0}^{\infty} \mathrm{d} t \int_{\mathbb{R}^{3}} u_{j}(x, t) \frac{\partial u_{i}(x, t)}{\partial x_{j}} \mathrm{~d} x= \\
& =\nu \int_{0}^{\infty} \mathrm{d} t \sum_{j=1}^{3} \int_{\mathbb{R}^{3}} \frac{\partial^{2} u_{i}(x, t)}{\partial x_{j}^{2}} \mathrm{~d} x-\int_{0}^{\infty} \mathrm{d} t \int_{\mathbb{R}^{3}} \frac{\partial p(x, t)}{\partial x_{i}} \mathrm{~d} x+ \\
& +\int_{0}^{\infty} \mathrm{d} t \int_{\mathbb{R}^{3}} f_{i}(x, t) \mathrm{d} x
\end{aligned}
$$

for $i=1,2,3$. Now by using (3), (6) and (7) in this equation, we have

$$
\int_{\mathbb{R}^{3}} \mathrm{~d} x\left[\int_{0}^{\infty} f_{i}(x, t) \mathrm{d} t+u_{i}^{0}(x)\right]=0 .
$$

Theorem 2.2. If there exists an index $i(1 \leq i \leq 3)$ such that

$$
\int_{\mathbb{R}^{3}} \mathrm{~d} x \int_{0}^{\infty}\left[u_{i}(x, t)+t f_{i}(x, t)\right] \mathrm{d} t \neq 0
$$

then the problem $C$ holds. 
Proof. First we multiple the equation (1) by $t$ and then we integrate it in the following form

$$
\begin{aligned}
& \int_{\mathbb{R}^{3}} \mathrm{~d} x \int_{0}^{\infty} t \frac{\partial u_{i}(x, t)}{\partial t} \mathrm{~d} t+\sum_{j=1}^{3} \int_{0}^{\infty} t \mathrm{~d} t \int_{\mathbb{R}^{3}} u_{j}(x, t) \frac{\partial u_{i}(x, t)}{\partial x_{j}} \mathrm{~d} x= \\
& \nu \sum_{j=1}^{3} \int_{0}^{\infty} t \mathrm{~d} t \int_{\mathbb{R}^{3}} \frac{\partial^{2} u_{i}(x, t)}{\partial x_{j}^{2}} \mathrm{~d} x-\int_{0}^{\infty} t \mathrm{~d} t \int_{\mathbb{R}^{3}} \frac{\partial p(x, t)}{\partial x_{i}} \mathrm{~d} x+ \\
&+\int_{0}^{\infty} t \mathrm{~d} t \int_{\mathbb{R}^{3}} f_{i}(x, t) \mathrm{d} x,
\end{aligned}
$$

for $i=1,2,3$. Now by integrating part by part and simplifying of the result, we obtain

$$
\int_{\mathbb{R}^{3}} \mathrm{~d} x \int_{0}^{\infty}\left[u_{i}(x, t)+t f_{i}(x, t)\right] \mathrm{d} t=0 .
$$

Theorem 2.3. If there exist indexes $i, k(1 \leq i, k \leq 3)$ such that

$$
\begin{gathered}
-\int_{\mathbb{R}^{3}} x_{k} u_{i}^{0}(x) \mathrm{d} x-\int_{0}^{\infty} \mathrm{d} t \int_{\mathbb{R}^{3}} u_{k}(x, t) u_{i}(x, t) \mathrm{d} x \neq \\
\delta_{i k} \int_{0}^{\infty} \mathrm{d} t \int_{\mathbb{R}^{3}} p(x, t) \mathrm{d} x+\int_{0}^{\infty} \mathrm{d} t \int_{\mathbb{R}^{3}} x_{k} f_{i}(x, t) \mathrm{d} x,
\end{gathered}
$$

then the problem $C$ holds.

Proof. First we multiple the equation (1) by $x_{k}(k=1,2,3)$ and then we integrate the result in the following form

$$
\begin{aligned}
& \int_{\mathbb{R}^{3}} x_{k} \mathrm{~d} x \int_{0}^{\infty} \frac{\partial u_{i}(x, t)}{\partial t} \mathrm{~d} t+\sum_{j=1}^{3} \int_{0}^{\infty} \mathrm{d} t \int_{\mathbf{R}^{3}} x_{k} u_{j}(x, t) \frac{\partial u_{i}(x, t)}{\partial x_{j}} \mathrm{~d} x= \\
&=\nu \sum_{j=1}^{3} \int_{0}^{\infty} \mathrm{d} t \int_{\mathbb{R}^{3}} x_{k} \frac{\partial^{2} u_{i}(x, t)}{\partial x_{j}^{2}} \mathrm{~d} x-\int_{0}^{\infty} \mathrm{d} t \int_{\mathbb{R}^{3}} x_{k} \frac{\partial p(x, t)}{\partial x_{i}} \mathrm{~d} x+ \\
&+\int_{0}^{\infty} \mathrm{d} t \int_{\mathbb{R}^{3}} x_{k} f_{i}(x, t) \mathrm{d} x,
\end{aligned}
$$

for $i=1,2,3$. Now by integrating part by part and simplifying of this equation, we have

$$
\begin{array}{r}
\quad-\int_{\mathbb{R}^{3}} x_{k} u_{i}^{0}(x) \mathrm{d} x-\int_{0}^{\infty} \mathrm{d} t \int_{\mathbb{R}^{3}} u_{k}(x, t) u_{i}(x, t) \mathrm{d} x= \\
=\delta_{i k} \int_{0}^{\infty} \mathrm{d} t \int_{\mathbb{R}^{3}} p(x, t) \mathrm{d} x+\int_{0}^{\infty} \mathrm{d} t \int_{\mathbb{R}^{3}} x_{k} f_{i}(x, t) \mathrm{d} x .
\end{array}
$$


Theorem 2.4. If there exists an index $i(1 \leq i \leq 3)$ such that

$$
\left.\int_{\mathbb{R}^{3}} \mathrm{~d} x \int_{0}^{\infty} t f_{i}(x, t)\right) \mathrm{d} t \neq 0,
$$

then the problem $C$ holds.

Proof. First multiple the equation (2) by $x_{k}(k=1,2,3)$ and then integrate it in the following form

$$
\sum_{i=1}^{3} \int_{0}^{\infty} \mathrm{d} t \int_{\mathbb{R}^{3}} x_{k} \frac{\partial u_{i}(x, t)}{\partial x_{i}} \mathrm{~d} x=0 .
$$

Hence,

$(* * *)$

$$
\int_{0}^{\infty} \mathrm{d} t \int_{\mathbb{R}^{3}} u_{k}(x, t) \mathrm{d} x=0,
$$

for $k=1,2,3$. Again, we can obtain the equation $(*)$ in a similar manner. Now by replacement $(* * *)$ in $(*)$, we have

$$
\int_{\mathbb{R}^{3}} \mathrm{~d} x \int_{0}^{\infty} t f_{i}(x, t) \mathrm{d} t=0 .
$$

Theorem 2.5. If there exist indexes $i, k$ with $1 \leq i<k \leq 3$, such that

$$
\int_{\mathbb{R}^{3}}\left[x_{i} u_{k}^{0}(x)-x_{k} u_{i}^{0}(x)\right] \mathrm{d} x \neq \int_{0}^{\infty} \mathrm{d} t \int_{\mathbf{R}^{3}}\left[x_{k} f_{i}(x, t)-x_{i} f_{k}(x, t)\right] \mathrm{d} x,
$$

then the problem $C$ holds.

Proof. For distinct indexes $i$ and $k(1 \leq i, k \leq 3)$ in $(* *)$, we have

$$
-\int_{\mathbb{R}^{3}} x_{k} u_{i}^{0}(x) \mathrm{d} x-\int_{0}^{\infty} \mathrm{d} t \int_{\mathbb{R}^{3}} u_{k}(x, t) u_{i}(x, t) \mathrm{d} x=\int_{0}^{\infty} \mathrm{d} t \int_{\mathbb{R}^{3}} x_{k} f_{i}(x, t) \mathrm{d} x .
$$

By replacement the indexes $i$ and $k$ in this equation, we obtain

$$
-\int_{\mathbb{R}^{3}} x_{i} u_{k}^{0}(x) \mathrm{d} x-\int_{0}^{\infty} \mathrm{d} t \int_{\mathbb{R}^{3}} u_{i}(x, t) u_{k}(x, t) \mathrm{d} x=\int_{0}^{\infty} \mathrm{d} t \int_{\mathbb{R}^{3}} x_{i} f_{k}(x, t) \mathrm{d} x .
$$

By subtracting of these equations, we have

$$
\int_{\mathbb{R}^{3}}\left[x_{i} u_{k}^{0}(x)-x_{k} u_{i}^{0}(x)\right] \mathrm{d} x=\int_{0}^{\infty} \mathrm{d} t \int_{\mathbb{R}^{3}}\left[x_{k} f_{i}(x, t)-x_{i} f_{k}(x, t)\right] \mathrm{d} x .
$$

Proposition 2.6. If for $k=1$ or $k=2$,

$$
\begin{gathered}
\int_{\mathbb{R}^{3}}\left[x_{3} u_{3}^{0}(x)-x_{k} u_{k}^{0}(x)\right] \mathrm{d} x+\int_{0}^{\infty} \mathrm{d} t \int_{\mathbb{R}^{3}}\left[u_{3}^{2}(x, t)-u_{k}^{2}(x, t)\right] \mathrm{d} x \neq \\
\int_{0}^{\infty} \mathrm{d} t \int_{\mathbb{R}^{3}}\left[x_{k} f_{k}(x, t)-x_{3} f_{3}(x, t)\right] \mathrm{d} x,
\end{gathered}
$$

then the problem $C$ holds. 
Proof. If the indexes $i, k$ are equal in $(* *)$, we have

$$
\begin{gathered}
-\int_{\mathbb{R}^{3}} x_{k} u_{k}^{0}(x) \mathrm{d} x-\int_{0}^{\infty} \mathrm{d} t \int_{\mathbb{R}^{3}} u_{k}^{2}(x, t) \mathrm{d} x= \\
\int_{0}^{\infty} \mathrm{d} t \int_{\mathbb{R}^{3}} p(x, t) \mathrm{d} x+\int_{0}^{\infty} \mathrm{d} t \int_{\mathbb{R}^{3}} x_{k} f_{k}(x, t) \mathrm{d} x .
\end{gathered}
$$

If we consider this equation for $k=3$ and subtract it form the results for $k=1$ and $k=2$, we obtain

$$
\begin{gathered}
\int_{\mathbb{R}^{3}}\left[x_{3} u_{3}^{0}(x)-x_{k} u_{k}^{0}(x)\right] \mathrm{d} x+\int_{0}^{\infty} \mathrm{d} t \int_{\mathbb{R}^{3}}\left[u_{3}^{2}(x, t)-u_{k}^{2}(x, t)\right] \mathrm{d} x= \\
\mathrm{d} s \int_{0}^{\infty} \mathrm{d} t \int_{\mathbb{R}^{3}}\left[x_{k} f_{k}(x, t)-x_{3} f_{3}(x, t)\right] \mathrm{d} x
\end{gathered}
$$

for $k=1,2$.

Theorem 2.7. Let $\varphi(x, t)$ be a function with continuous first order partial derivatives such that

$$
\sum_{i=1}^{3} \int_{\mathbb{R}^{3}} u_{i}(x, t) \frac{\partial \varphi(x, t)}{\partial x_{i}} \mathrm{~d} x \neq 0
$$

for all $t>0$. Then, the problem $C$ holds.

Proof. First multiple the equation $(2)$ by $\varphi(x, t)$ and then integrate it. By simplification of the result, we obtain

$$
\sum_{i=1}^{3} \int_{\mathbb{R}^{3}} u_{i}(x, t) \frac{\partial \varphi(x, t)}{\partial x_{i}} \mathrm{~d} x=0,
$$

for all $t>0$.

\section{The Problem D}

In this section we shall give some sufficient conditions for non-existence of solutions of the problem D.

Theorem 3.1. If there exists an index $i(1 \leq i \leq 3)$ such that

$$
\int_{[0,1]^{3}}\left[\int_{0}^{\infty} f_{i}(x, t) \mathrm{d} t+u_{i}^{0}(x)\right] \mathrm{d} x \neq 0,
$$

then the problem $D$ holds.

Proof. The proof is based on the proof of Theorem 2.1, by replacing integration in $x$ on $\mathbb{R}^{3}$ with the one on $[0,1]^{3}$.

Theorem 3.2. If there exists an index $i(1 \leq i \leq 3)$ such that

$$
\int_{[0,1]^{3}} \mathrm{~d} x \int_{0}^{\infty}\left[u_{i}(x, t)+t f_{i}(x, t)\right] \mathrm{d} t \neq 0,
$$

then the problem $D$ holds. 
Proof. The proof is based on the proof of Theorem 2.2, by replacing integration in $x$ on $\mathbf{R}^{3}$ with the one on $[0,1]^{3}$.

Theorem 3.3. Let $\varphi(x, t)$ be a function with continuous first order partial derivatives such that

$$
\sum_{i=1}^{3} \int_{[0,1]^{3}} u_{i}(x, t) \frac{\partial \varphi(x, t)}{\partial x_{i}} \mathrm{~d} x \neq 0
$$

for all $t>0$. Then, the problem $D$ holds.

Proof. The proof is based on the proof of Theorem 2.7, by replacing integration in $x$ on $\mathbb{R}^{3}$ with the one on $[0,1]^{3}$.

\title{
REFERENCES
}

[1] N. Aliev, M. Jahanshahi, S.M. Hosseini, An Analytic-Numerical Method for 3-dimensional Steady-state Navier-Stokes Equations II, Int. J. Differ. Equ. Appl., 10 (2005), no. 2, 135-145.

[2] N. Aliev, S.M. Hosseini, Cauchy Problem for the Navier-Stokes Equation and its Reduction to a Non-linear System of Second Kind Fredholm Integral Equations, Int. J. Pure and Appl. Math., Vol. 3, no. 3 (2002), 317-324.

[3] P. Constantin, C. Fefferman, Direction of vorticity and the problem of global regularity for the Navier-Stokes equations, Indiana Univ. Math. J., 42 (1993), no. 3, 775-789.

[4] C.L. Fefferman, Existence and Smoothness of the Navier-Stokes Equation, to appear.

[5] N. Vlachakis, D. Pavlou, V. Vlachakis, M. Pavlou, M. Kouskouti, An exact solution of NavierStokes equations for the flow through a diverging artery. Advances in fluid mechanics, Adv. Fluid Mech., 40, WIT Press, Southampton, 2004, 389-394.

\author{
Sh. REZAPOUR \\ Department of Mathematics \\ Azarbaidjan University of Tarbiat Moallem \\ AZARSHAHR, TABRIZ \\ IRAN \\ E-mail address: sh.rezapour@azaruniv.edu
}

M. JAHANSHAHI

E-mail address: jahan_m15@yahoo.com 